\title{
Recombinant Ganoderma lucidum immunomodulatory protein modified with polyethylene glycol
}

\author{
XIPING ZHANG, FEI SUN, ZHIYI LIU, SHUQIN ZHANG and CHONGYANG LIANG \\ Institute of Frontier Medical Science, Jilin University, Changchun 130021, P.R. China
}

Received October 16, 2012; Accepted January 16, 2013

DOI: $10.3892 / \mathrm{mmr} .2013 .1281$

\begin{abstract}
Recombinant Ganoderma lucidum immunomodulatory protein (rLZ-8) expressed using the Pichia yeast eukaryotic expression system is a potential new drug for cancer therapy; however, it has a short half-life in the body. In order to optimize the potency and stability of rLZ-8, we modified the recombinant protein chemically using methoxyPEG-succinimidyl propionate (mPEG-SPA). The results indicated that several parameters, including $\mathrm{pH}$, the molar ratio of rLZ-8 to mPEG-SPA and time, played crucial roles in the modification process. In particular, when the molar ratio of rLZ- 8 to mPEG-SPA was $1: 1$, rLZ-8 was modified by a single mPEG-SPA moiety. In addition, MALDI-TOF/TOF and ESI Q-Trap results revealed that the difference in molecular weight (MW) between the peptide-linked mPEG-SPA and the mPEG-SPA closely matched the MW of a methionine amino acid. Taken together, these data suggest that modification of mPEG-SPA occurred on the N-terminal helix of rLZ-8. This modification method has laid a foundation for the development of long-acting formulations of rLZ-8.
\end{abstract}

\section{Introduction}

Recombinant Ganoderma lucidum immunomodulatory protein (rLZ-8), expressed using a Pichia yeast eukaryotic expression system (1-3), was the first member of the fungal immunomodulatory protein (FIP) family discovered in mushrooms. It has been shown that rLZ-8 enjoys a promising prospect as a new class I drug, since it induces apoptosis in K562 and HL60 cells and acts as an immunomodulating protein in a variety diseases, including in the non-obese diabetes mouse model and in immunomodulation of foodinduced allergic reactions (4-14). However, rLZ-8 has a MW

Correspondence to: Professor Chongyang Liang, Department of Cell Biology, Institute of Frontier Medical Science, Jilin University, 1266 Fujin Road, Changchun 130021, P.R. China

E-mail: liang@jlu.edu.cn

Key words: recombinant Ganoderma lucidum immunomodulatory protein, PEGylated succinimidyl propionate, gel filtration chromatography, isolation and purification, modification locus confirmation of only $13 \mathrm{kDa}$ and its dynamic properties, such as the halflife, are not satisfactory, which enormously limits its clinical application (15-19).

In this study, we explored a strategy to overcome the natural shortcomings of rLZ- 8 using conventional PEGylation technology to prolong its half-life and to enhance its stability (20-27). We optimized the modification and purification process, successfully prepared the single-point modification product and carried out an initial characterization of the association between the modification sites and the activity of the modified product. This study has provided insights into the structure-activity relationship of rLZ-8 and has also laid a foundation for the development of long-acting formulations of rLZ-8.

\section{Materials and methods}

Instruments and reagents. The following instruments/ instrument systems were used in this study: HPLC System (Shimadzu, Japan); AKTA Explorer 100 protein purification workstation (GE Healthcare, Franklin Lakes, NJ, USA); ultrafiltration cup (GE Healthcare); gel imager (Tanon, Shanghai, China); SDS-PAGE electrophoresis system (GE Healthcare); electrospray ionization source high-resolution tandem mass spectrometer (ESI-Q/TOF, Applied Biosciences, Carlsbad, CA, USA) and laser desorption time-of-flight mass spectrometry (MALDI-TOF/TOF, Applied Biosciences); Hiload 16/70 Superdex $^{\mathrm{TM}} 75$ gel column (GE Healthcare). Methoxy-PEGsuccinimidyl propionate mPEG-SPA $(5 \mathrm{kDa})$ was purchased from YareBio (Shanghai, China), and the recombinant Ganoderma lucidum immunomodulatory protein (rLZ-8) was obtained from our laboratory.

\section{Modification optimization}

Method. Optimization of the output of the single-point modification reaction was carried out by altering one single effect factor and keeping other factors the same in multi-level repeated trials. The effect of each factor on the conformation of reaction products was analyzed using SDS-PAGE and highperformance liquid chromatography (HPLC).

Effect of $p H$ and ionic strength. The modification of rLZ-8 with $\mathrm{mPEG}-\mathrm{SPA}(1: 12$, mol:mol) was carried out in a series of buffer systems at varying $\mathrm{pH}$, namely $\mathrm{pH} 4.0,5.0,6.0,7.0$ or 8.0 , and then in $\mathrm{pH} 8.0$ phosphate buffer with varying ionic strengths, at $0.025,0.05,0.1$ or $0.2 \mathrm{M}$, at room temperature for 


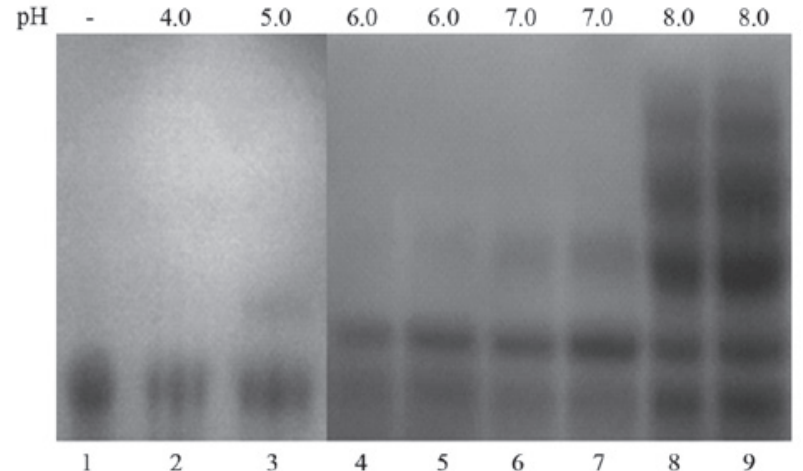

Figure 1. SDS-PAGE of PEGylation reaction mixture, with the reaction performed with phosphate buffers of the indicated $\mathrm{pH}$. Lanes: 1, mPEG-SPA alone; 2 , reaction mixture in $\mathrm{pH} 4.0 ; 3$, reaction mixture in $\mathrm{pH} 5.0 ; 4$ and 5 , reaction mixture in $\mathrm{pH}$ 6.0; 6 and 7, reaction mixture in $\mathrm{pH} 7.0 ; 8$ and 9, reaction mixture in $\mathrm{pH} 8.0$.

$2 \mathrm{~h}$, away from light. The results were analyzed by SDS-PAGE followed by gel imaging after staining with barium iodide and Coomassie Brilliant Blue.

Effect of reaction time and temperature. In order to address the effect of time and temperature on the modification reaction, rLZ-8 and mPEG-SPA (1:12, mol:mol) were incubated together in a phosphate buffer system $(0.1 \mathrm{M}, \mathrm{pH} 8.0)$ at 4,16 , 25 and $37^{\circ} \mathrm{C}$, and sampled every $0.5 \mathrm{~h}$ for analysis by HPLC, up to $2.5 \mathrm{~h}$.

Effect of molar ratio of $r L Z-8$ and $m P E G-S P A$. rLZ-8 and mPEG-SPA in a phosphate buffer system $(0.1 \mathrm{M}, \mathrm{pH} 8.0)$ at molar ratios of 1:1, 1:3, 1:6, 1:12 and 1:24, were mixed at room temperature for $2 \mathrm{~h}$, away from light. HPLC and SDS-PAGE with barium iodide or Coomassie Brilliant Blue staining were used to monitor the reaction products.
Purification and identification of the desired target product. The target product was purified using a Hiload 16/70 Superdex 75 gel chromatographic column with $0.05 \mathrm{M}$ phosphate buffer containing $0.15 \mathrm{M} \mathrm{NaCl}(\mathrm{pH} 7.0)$ and a flow rate of $1 \mathrm{ml} / \mathrm{min}$. After equilibrating the column with the phosphate buffer for 2 column volumes $(120 \mathrm{ml})$, iso-concentration elution was applied for another 1.5 column volumes, with peak collection at every $2 \mathrm{ml}$ and detection wavelengths of 280,254 and $215 \mathrm{~nm}$. Every peak was detected using SDS-PAGE and HPLC and the retention times of the confirmed peaks were recorded.

Measurement of the biological activity of the target product. A lymphocyte transformation test (BrdU reagent box) was performed according to the manufacturer's instructions in order to determine the biological activity of rLZ-8 and mPEG-SPA-rLZ-8. The lymphocyte transformation test is based on the fact that lymphocytes stimulated by certain antigens transform and proliferate when re-exposed to the same antigens. The biological activity of antigens is measured by monitoring the proliferation of stimulated cells, and by measuring the incorporation of BrdU into replicating DNA. A chromophore coupled to an anti-BrdU antibody enables spectrophotometric detection of proliferating cells.

\section{Analysis of the initial characteristics of the target product}

Detection of protein content. We used the Lowry method to measure the protein concentration of the reaction product according to the guidelines of the Chinese Pharmacopoeia 2010 Appendix VIB.

Validation of molecular weight and modification site. Firstly, the sample (in the form of dry powder) was placed into $50 \mathrm{mM} \mathrm{NH}_{4} \mathrm{HCO}$ to prepare a $1 \mathrm{mg} / \mathrm{ml}$ sample solution. A 20- $\mu 1$ aliquot of the sample solution was then diluted to obtain a $10-\mathrm{mM}$ solution by adding $100 \mathrm{mM}$ dithiothreitol
A
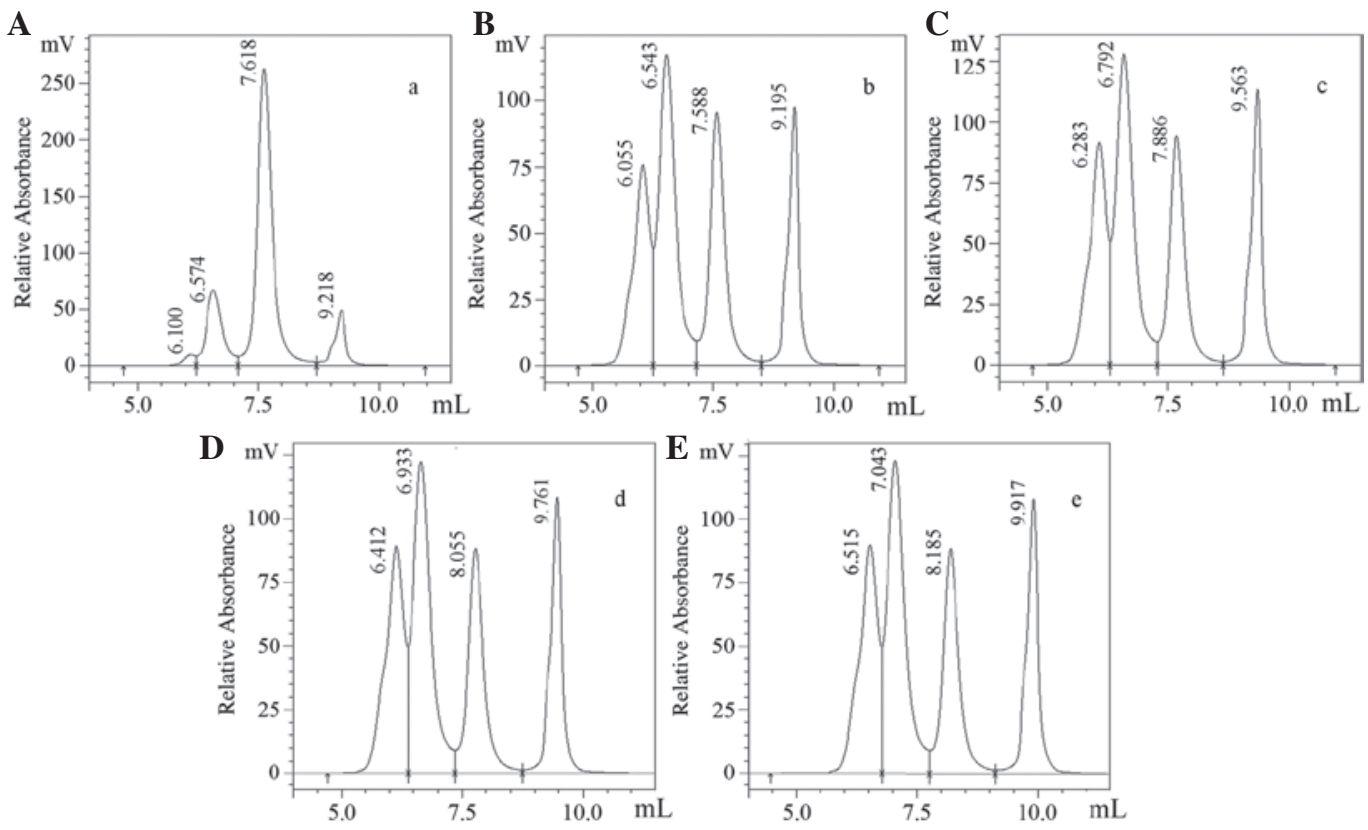

Figure 2. Size exclusion chromatography (SEC)-high-performance liquid chromatography (HPLC) analysis of the modification reaction mixture at (A) 0.5 h, (B) $1 \mathrm{~h},(\mathrm{C}) 1.5 \mathrm{~h},(\mathrm{D}) 2 \mathrm{~h}$ and (E) $2.5 \mathrm{~h}$ after incubation at room temperature. 


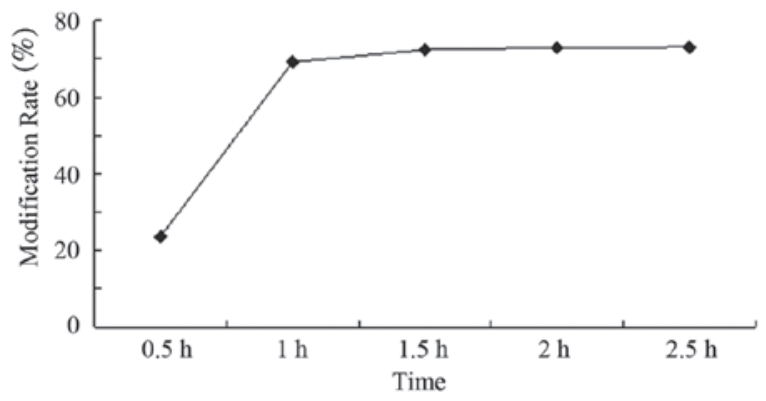

Figure 3. Rate of modification of rLZ-8 with mPEG-SPA at various time points.

(DTT) and heating at $56^{\circ} \mathrm{C}$ for $1 \mathrm{~h}$. After cooling the mixture to room temperature, $250 \mathrm{mM}$ indole-3-acetic acid (IAA) was added to obtain a $25-\mathrm{mM}$ sample solution and this mixture was protected from light for $1 \mathrm{~h}$. After completion of this step, $0.5 \mu \mathrm{g}$ Trypsin was added and the sample was digested at $37^{\circ} \mathrm{C}$ for $12 \mathrm{~h}$. Finally, the reaction was ended by adding $1 \mu \mathrm{l}$ of $10 \%$ trifluoroacetic acid (TFA). The digestion products and matrix were mixed at a proportion of 1:3 and dried atmospherically. The peptide mass was detected by PMF analysis in order to identify the PEGylated site on $\mathrm{rLZ}-8$ in the following modes: the mass spectra for the $500-4000 \mathrm{~m} / \mathrm{z}$ range were determined in the reflection positive ion mode and the $1000-10000 \mathrm{~m} / \mathrm{z}$ range was determined in the linear positive ion mode.

\section{Results}

\section{Modification optimization}

Effect of $\mathrm{pH}$ and ionic strength. The results of SDS-PAGE analysis of the PEGylation reaction mixture following barium iodide staining are shown in Fig. 1. As the figure indicates, no modification reaction (indicated by the appearance of higher-MW bands) occurred until pH 5.0, and a weak reaction occurred between $\mathrm{pH} 5.0$ and 7.0. When the $\mathrm{pH}$ was $>8$, the reaction was more efficient and numerous modification products were detected. By contrast, variation of ionic strength

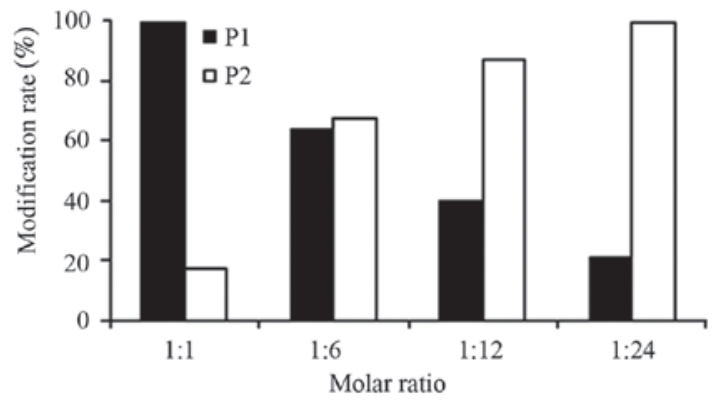

Figure 5. Histogram of the modification rate of reaction mixtures with various molar ratios of rLZ-8 to mPEG-SPA. P1, percent of mono-mPEGmodified rLZ-8 in the modification mixture; P2, percent of mPEG-modified rLZ-8 in the modification mixture.

hardly affected the modification reaction. Therefore, we chose $\mathrm{pH} 8.0$ as the reaction $\mathrm{pH}$ in further studies.

Effect of reaction time and temperature. The reaction products were single and relatively unstable at $0.5 \mathrm{~h}$, which indicates the reaction was still in progress (Fig. 2A). Between 1 and $2.5 \mathrm{~h}$, the reaction results appeared to be similar (as shown in Fig. 2B-E) and the reaction products did not change significantly (Fig. 3), indicating that the reaction had reached equilibrium after $1 \mathrm{~h}$. Additionally, the experiment was undertaken at room temperature, since temperature did not have a significant effect on the reaction products (data not shown). As a result, we chose room temperature and $2 \mathrm{~h}$ as the reaction conditions.

Effect of molar ratio of $r L Z-8$ and $m P E G-S P A$. Fig. 4 illustrates how the molar ratios of rLZ-8 to mPEG-SPA influenced the modification reaction. The modification products became increasingly varied (as indicated by the less discrete appearance of the higher-MW bands) when the molar ratio of rLZ-8 to mPEG-SPA was decreased. Only one type of modification product occurred at the molar ratio of 1:1, so each modification product occupies $100 \%$ of the product, with the exception of the remaining unmodified $\mathrm{rLZ}-8$. When the molar ratio was $1: 24$, there were four modification products and each one contributed to $18.57 \%$ of the total mixture (Figs. 4 and 5). For
A $\mathrm{kDa}$

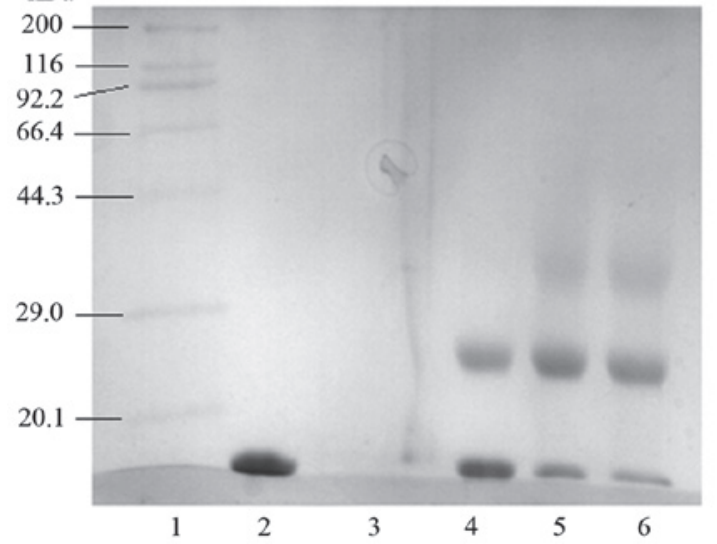

B

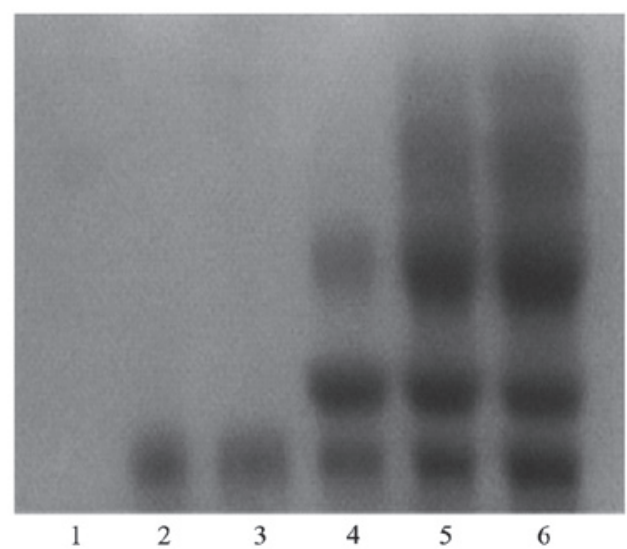

Figure 4. SDS-PAGE analysis of the modification reaction at various molar ratios of rLZ-8 to mPEG-SPA. (A) Barium iodide staining of the SDS-PAGE gel. (B) Coomassie Brilliant Blue staining of the SDS-PAGE gel. Lanes: 1, MW marker; 2, rLZ-8; 3-6, molar ratio of rLZ-8 to mPEG-SPA in the reaction mixture was $1: 1,1: 6,1: 12$ and $1: 24$, respectively. 
A maU

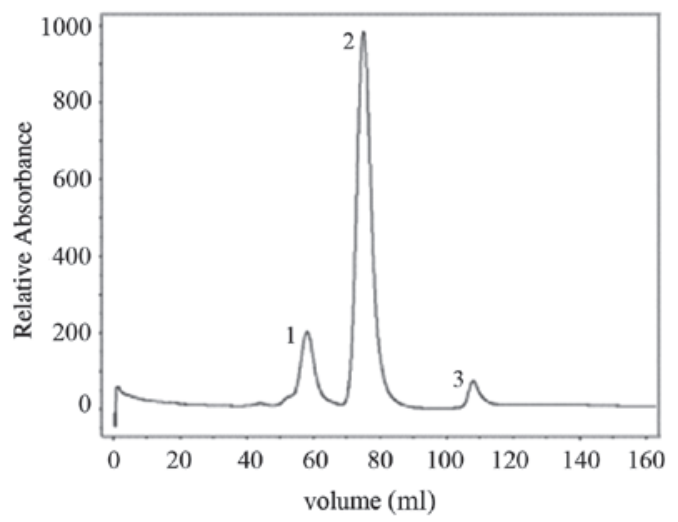

B $\mathrm{mV}$

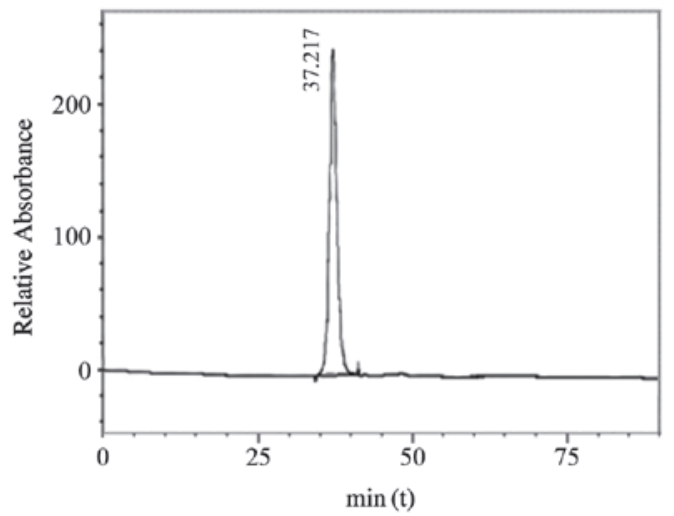

Figure 6. Preparation and purification of mPEG-SPA-rLZ-8 with size exclusion chromatography (SEC). (A) Purification of modification reaction mixture by Superdex 75 (1, target protein; 2, rLZ-8; 3, by-products); (B) High-performance liquid chromatography (HPLC) of target protein after purification.

A $\mathrm{kDa}$

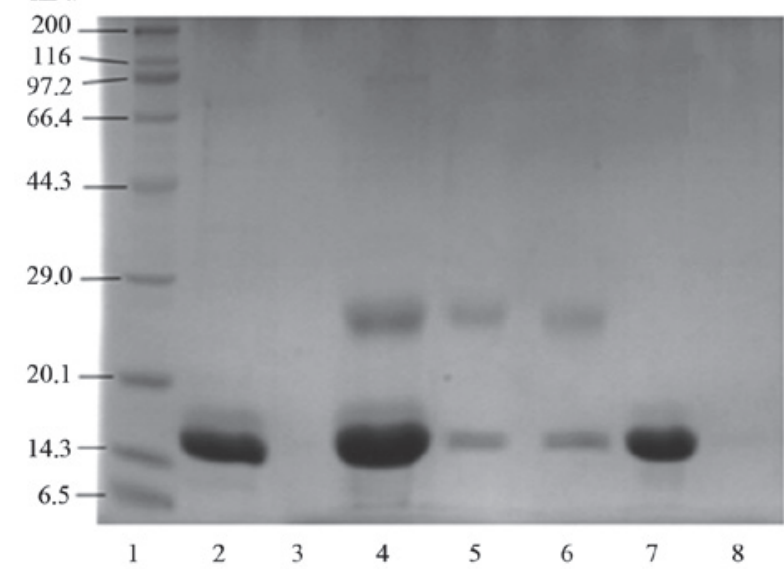

B

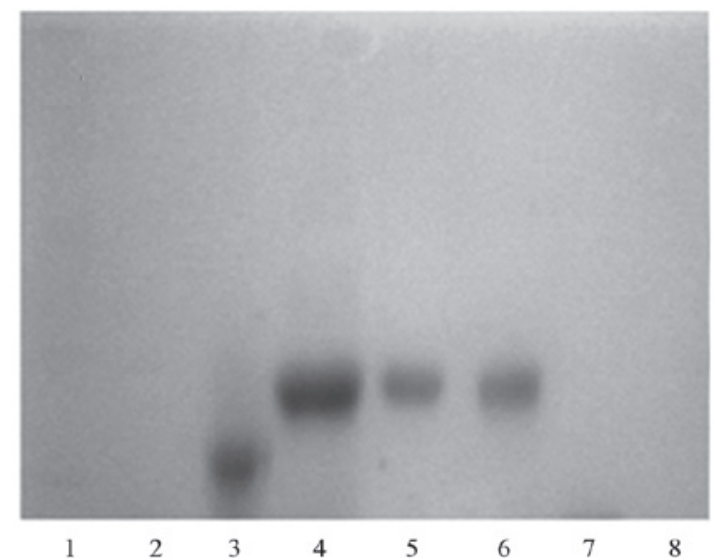

Figure 7. SDS-PAGE analysis of various absorption peaks by Superdex 75 purification. (A) Barium iodide staining of SDS-PAGE gel. (B) Coomassie Brilliant Blue staining of SDS-PAGE gel. Lanes: 1, MW marker; 2, rLZ-8; 3, mPEG-SPA; 4, modification mixture; 5, the first half of peak 1 (shown in Fig. 6A); 6, the last half of peak 1 (shown in Fig. 6A); 7, peak 2 (shown in Fig. 6A); 8, peak 3 (shown in Fig. 6A).

ease of purification, a molar ratio of 1:1 was chosen for singlepoint modification.

Purification and identification of the target product. As shown in Fig. 6A and Fig. 7, only one type of modification product occurred at a molar ratio of 1:1. According to the molecular sieve principle, the purity of the purification product may reach $99 \%$ after being purified using a Superdex 75 gel chromatographic column, a result that we also observed when using this chromatographic purification method (Fig. 6B).

Biological activity of the purified target product. The biological activities of rLZ-8 and PEG-rLZ-8 (as assessed using a BrdU incorporation assay) are shown in Fig. 8. When modified by PEG, the activity of the modification products of rLZ-8 decreased as expected.

Preliminary analysis of the characteristics of target products Detection of protein content. The Lowry method was used to determine the concentration of protein in the modification product due to its outstanding linear correlation, both before and after modification. The amount of rLZ-8 decreased from

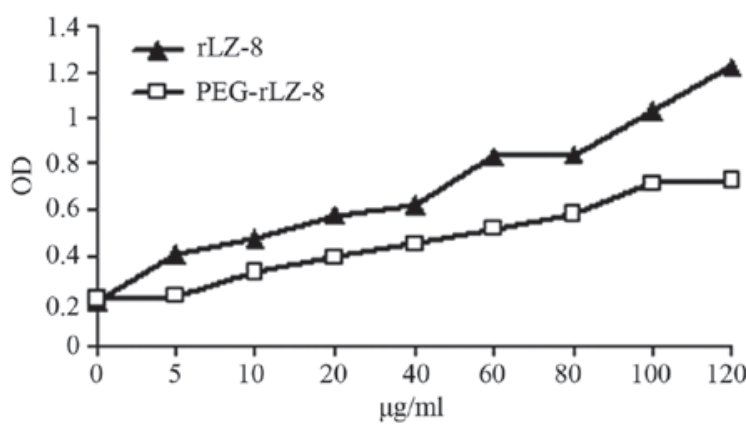

Figure 8. BrdU assay for determining the biological activity of rLZ-8 and PEG-rLZ-8. The incorporation of BrdU was measured in the lymphocyte transformation test using rLZ-8 (ム) and PEG-rLZ-8 ( $\square$ ) as the antigens. The plotted optical density (OD) value is indicative of the amount of BrdU incorporated into replicating DNA in the stimulated lymphocytes.

5 to $0.96 \mathrm{mg}$ following the modification and purification process.

Validation of molecular weight and modification site. Several points were considered in order to correctly identify the modification site. Firstly, the PEG-peptide may not match 

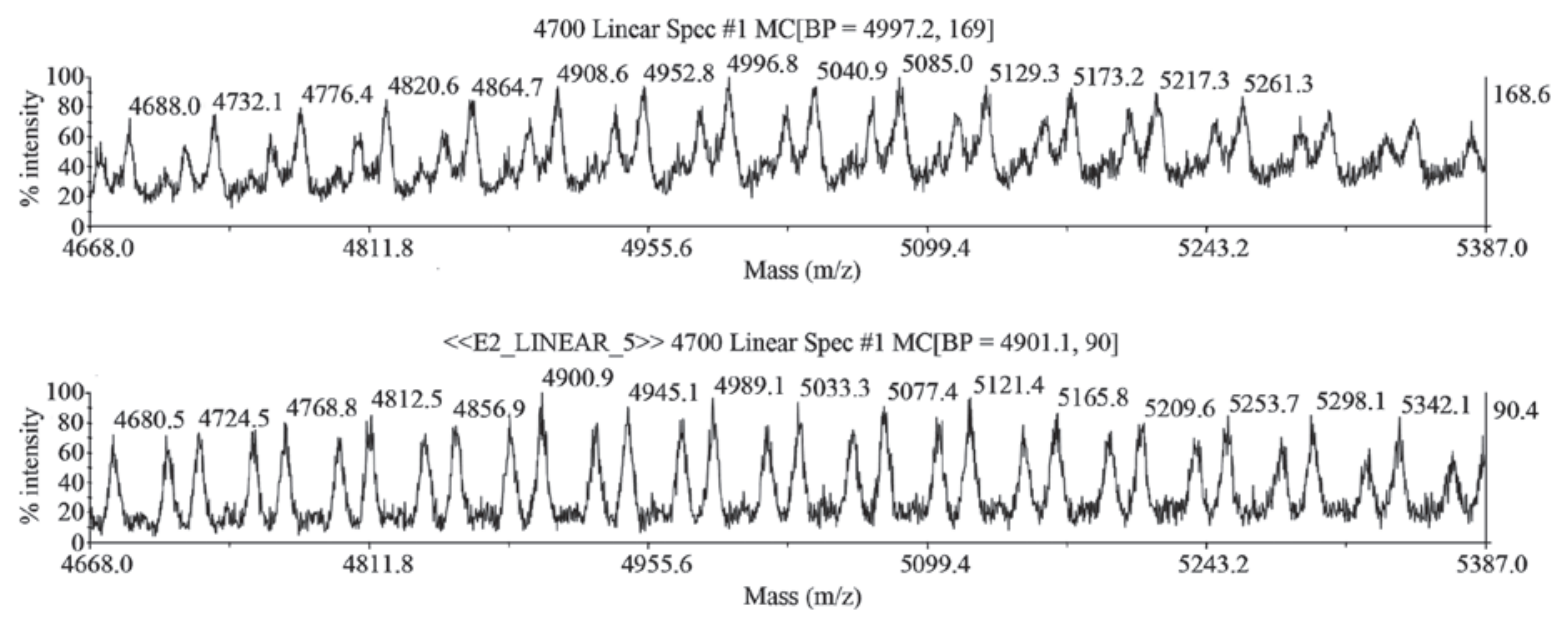

Figure 9. MS/MS analysis of PEG and PEG-peptide molecular weight.

the expected peptide mass fingerprint (PMF) and those that match usually represent unPEGylated peptides in the modification sample. Secondly, the PEGylated site is always located at the N-terminus or on a Lys side chain. Thirdly, PEGylated Lys is usually intractable to enzymatic cleavage, in which case, when Lys is modified at least one uncleaved site is always included, and the margin between the PEG-peptide and PEG should be close to the theoretical MW of the peptide, while the MS/MS peak shape of the PEG-peptide should be consistent with that of PEG.

From the data, we conclude that Lys is not the PEGylated site, as all Lys in the PEG-protein matched the PMF and the molecular weight of the PEG-peptides were close to that of PEG. Therefore, we concluded that the PEGylated site did not fall within the 75-111 amino acid fragment of rLZ-8 (this fragment is $4205.9858 \mathrm{Da}$ ). In the determination of the PMF of the PEG-protein, N-terminal peptides with and without the $\mathrm{M}$ fragment (SDTALIFR) were observed, so the PEGylated site is estimated to be at the N-terminus, as the falling off of $\mathrm{M}$ in the process is not expected to affect the correspondence in MW between the peptides and the PMF (Fig. 9). Furthermore, the margin of the MW between PEG-peptides and PEG are relatively close to $\mathrm{M}$, which provides further evidence that the PEGylated site is located at the N-terminus.

\section{Discussion}

In this study, we established a strategy for modifying rLZ-8 using $\mathrm{mPEG}$-SPA and evaluated the effect of various reaction parameters on the modification reaction. The $\mathrm{pH}$ of the reaction buffer was the determining factor in the modification reaction, and the modification reaction could not be carried out under acidic conditions. The desired reaction products were recovered in $\mathrm{pH} 8.0$ phosphate buffer solution; however, the molar ratio of rLZ-8 to mPEG-SPA was also an important factor affecting the reaction products. A molar ratio of 1:1 yielded a single-site modification product, and the composition of the reaction products became more and more complex as the amount of mPEG-SPA was increased. When the molar ratio was $>1: 12$, there were four species of products, of $\sim 66,44,30$ and $17.9 \mathrm{kDa}$. The reaction temperature had almost no effect on the modification reaction, and the reaction reached equilibrium after $1 \mathrm{~h}$. From these data, we concluded that the best conditions to obtain a single-site modified product are: $0.05 \mathrm{M}, \mathrm{pH} 8.0$ phosphate buffer solution, a 1:1 molar ratio of rLZ to $\mathrm{mPEG}-\mathrm{SPA}$, and stirring at room temperature for $2 \mathrm{~h}$, protected from light.

PEG modification sites are generally at the $\mathrm{N}$-terminus or on Lys side chains. The structural analysis of rLZ-8 (17) revealed that the active form of $\mathrm{rLZ}-8$ is a non-covalently bonded dimer and that each monomer contains 6 Lys. Therefore, in theory, one rLZ-8 molecule may combine with 1-7 mPEG-SPA molecules, but the mPEG can only modify the N-terminus of the protein. Since mPEG-SPA has long-chain branches and is sticky, the greater the amount of mPEG-SPA that combines with the protein, the greater the reduction in activity, and the more complex the product composition is likely to be. In this study, we focused on optimizing reaction conditions to obtain a single-site modified product.

The MW of the single-site modified product is $\sim 17.9 \mathrm{kDa}$, so we selected the Superdex 75 separation column to purify the modification reaction mixture. Purification results revealed that the modified products can be separated completely from the unreacted rLZ-8 and that the small molecule byproducts can be removed. Moreover, the single-site modified products were of high purity and the excess $\mathrm{rLZ}-8$ could be recycled and reused in the experiment. Separation and purification of a variety of modified products in excess of mPEG-SPA is more difficult, and further studies are required to optimize recovery of other modification products.

An in vitro assay for assessing the biological activity of the single-site modified product indicated that mPEG-SPA altered the efficacy of the protein in a lymphocyte transformation assay. Additional in vivo experiments should be carried out to determine the pharmacokinetic properties, such as the halflife and effective dose, of the modified rLZ-8. Such detailed analysis is likely to facilitate the clinical application of modified products such as PEGylated rLZ-8.

\section{Acknowledgements}

This study was supported by the Science and Technology Development Program of Jilin Province (Grant No. 20090941). 


\section{References}

1. Liang C, Zhang S, Liu Z and Sun F: Ganoderma lucidum immunomodulatory protein(Lz-8) expressed in Pichia pastoris and the identification of immunocompetence. Sheng Wu Gong Cheng Xue Bao 25: 441-447, 2009.

2. Lin JW, Hao LX, Xu GX, Sun F, Gao F, Zhang R and Liu LX: Molecular cloning and recombinant expression of a gene encoding a fungal immunomodulatory protein from Ganoderma lucidum in Pichia pastoris. World J Microbiol Biotechnol 25: 383-390, 2009.

3. Murasugi A, Tanaka S, Komiyama N, Iwata N, Kino K, Tsunoo H and Sakuma S: Molecular cloning of a cDNA and a gene encoding an immunomodulatory protein, Ling Zhi-8, from a fungus, Ganoderma lucidum. J Biol Chem 266: 2486-2493, 1991.

4. Wang XL, Liang CY, Li HR, Li BZ and Sun F: Recombinant Ganoderma lucidum immunoregulatory protein (rLZ-8) induces nuclear-stress apoptosis in K562 cells. Zhongguo Mian Yi Xue Za Zhi 26: 616-618, 2010 (In Chinese).

5. Guo Q, Sun H, Liang CY, Zhang SQ, Liu ZY and Sun F: Inhibiting and apoptosis-inducing effects of recombinant Ganoderma lucidum immunoregulatory protein on HL60 cells. Zhongguo Mian Yi Xue Za Zhi 26: 520-522, 2010 (In Chinese).

6. Liang CY, Zhang SQ and Sun F: The dynamic observation: cellular localization of fluorescein isothiocyanate labeled recombinant Ganoderma lucidum immunoregulatory protein (rLZ-8) in NB4 APL cell. Chemical Journal of Chinese Universities: CJCU 30: 479-483, 2009.

7. Kino K, Mizumoto K, Sone T, Yamaji T, Watanabe J, Yamashita A, Yamaoka K, Shimizu K, Ko K and Tsunoo H: An immunomodulating protein, Ling Zhi-8 (LZ-8) prevents insulitis in non-obese diabetic mice. Diabetologia 33: 713-718, 1990.

8. Haak-Frendscho M, Kino K, Sone T and Jardieu P: Ling Zhi-8: a novel T cell mitogen induces cytokine production and upregulation of ICAM-1 expression. Cell Immunol 150: 101-113, 1993.

9. Hsieh KY, Hsu CI, Lin JY, Tsai CC and Lin RH: Oral administration of an edible-mushroom-derived protein inhibits the development of food-allergic reactions in mice. Clin Exp Allergy 33: 1595-1602, 2003.

10. Liu YH, Kao MC, Lai YL and Tsai JJ: Efficacy of local nasal immunotherapy for 10. Dp2-induced airway inflammation in mice: using Dp2 peptide and fungal immunomodulatory peptide. J Allergy Clin Immunol 112: 301-310, 2003.

11. Ho JC, Sze SC, Shen WZ and Liu WK: Mitogenic antivity of edible mushroom lectins. Biochim Biophys Acta 1671: 9-17, 2004.

12. Wasser SP and Weis AL: Therapeutic effects of substances occurring in higher Basidiomycetes mushrooms: a modern perspective. Crit Rev Immunol 19: 65-96, 1999.
13. Llovet JM and Bruix J: Systematic review of randomized trials for unresectable hepatocellular carcinoma: chemoembolization improves survival. Hepatology 37: 429-442, 2003.

14. Liang CY, Li H, Zhou H, Zhang SQ, Liu ZY, Zhou Q and Sun F: Recombinant Lz-8 from Ganoderma lucidum induces endoplasmic reticulum stress-mediated autophagic cell death in SGC-7901 human gastric cancer cells. Oncol Rep 27: 1079-1089, 2012.

15. van der Hem LG, van der Vliet JA, Bocken CF, Kino K, Hoitsma AJ and Tax WJ: Ling Zhi-8: studies of a new immunomodulating agent. Transplantation 60: 438-443, 1995.

16. Zhu JQ, Liang CY, Feng K, Gai XD, Sun X and Sun F: Purification and properties of recombinant Ganoderma lucidum immunoregulatory protein. Chem Res Chin Univ 29: 1-4, 2008.

17. Huang L, Sun F, Liang CY, He YX, Bao R, Liu L and Zhou CZ: Crystal structure of LZ-8 from the medicinal fungus Ganoderma lucidium. Proteins 75: 524-527, 2009.

18. Lin WH, Hung CH, Hsu CI and Lin JY: Dimerisation of the N-terminal amphipathic $\alpha$-helix domain of the fungal immunomodulatory protein from Ganoderma tsugae (Fip-gts) defined by a yeast two-hybrid system and site-directed mutagenesis. J Biol Chem 272: 20044-20048, 1997.

19. Tanaka S, Ko K, Kino K, Tsuchiya K, Yamashita A, Murasugi A, Sakuma $\mathrm{S}$ and Tsunoo $\mathrm{H}$ : Complete amino acid sequence of an immunomodulatory protein, ling zhi-8 (LZ-8). An immunomodulator from a fungus, Ganoderma lucidium, having similarity to immunoglobulin variable regions. J Biol Chem 264: 16372-16377, 1989.

20. Roberts MJ, Bentley MD and Harris JM: Chemistry for peptide and protein PEGylation. Adv Drug Deliv Rev 54: 459-476, 2002.

21. Fee CJ and Van Alstine JM: PEG-proteins: Reaction engineering and separation issues. Chem Eng Sci 61: 924-939, 2005.

22. Kinstler O, Molineux G, Treuheit M, Ladd D and Gegg C: MonoN-terminal poly(ethylene glycol)-protein conjugates. Adv Drug Deliv Rev 54: 477-485, 2002

23. Greenwald RB: PEG drugs: an overview. J Control Release 74: 159-171, 2001.

24. Seyfried BK, Siekmann J, Belgacem O, Wenzel RJ, Turecek PL and Allmaier G: MALDI linear TOF mass spectrometry of PEGylated (glyco)proteins. J Mass Spectrom 45: 612-617, 2010.

25. Ronda L, Pioselli B, Bruno S, Faggiano S and Mozzarelli A Electrophoretic analysis of PEGylated hemoglobin-based blood substitutes. Anal Biochem 408: 118-123, 2011.

26. Zhai Y, Zhao Y, Lei J, Su Z and Ma G: Enhanced circulation half-life of site-specific PEGylated rhG-CSF: optimization of PEG molecular weight. J Biotechnol 142: 259-266, 2009.

27. Moosmann A, Christel J, Boettinger H and Mueller E: Analytical and preparative separation of PEGylated lysozyme for the characterization of chromatography media. J Chromatogr A 1217: 209-215, 2010 